present in $4.1 \%$ (14) patients while in eight of these patients had complete resection with no evidence of recurrence at follow-up. Over all Complication rate was $9.1 \%$ (31). 6.5\% (22) had immediate bleeding requiring therapy. 15/22 were left sided Polyps (11 rectum, 4 sigmoid) with mean size $>20 \mathrm{~mm}$. $1.1 \%$ (4) patients had delayed bleeding. While two required repeat colonoscopy and haemostasis one had right hemicolectomy as bleeding was complicated by delayed perforation. $0.5 \%$ (2) patients had immediate while one patient had delayed perforation at second day requiring surgery. There was no procedure related death.

Conclusion EMR is effective and safe therapy in well trained hands with minor and acceptable complication rate. There is high recurrence rate especially after piecemeal resection within the first 12 months that requires a strict follow-up protocol. Rectal polyps and size $>20 \mathrm{~mm}$ were associated with high risk of bleeding. Currently there are no guidelines and standards measuring EMR outcome. Nationwide EMR audit/database is needed to help form recommendations.

Competing interests None declared.

\section{PWE-197 OPTIMISATION OF THE FICE TECHNIOUE FOR ENHANCEMENT OF OESOPHAGEAL PATHOLOGY}

doi:10.1136/gutjnl-2012-302514d.197

${ }^{1} S$ Inglis, ${ }^{2} S$ Alexandridis, ${ }^{2} \mathrm{~J} N$ Plevris. ${ }^{1}$ Department of Medical Physics, NHS Lothian, Edinburgh, UK; ${ }^{2}$ Centre for Liver \& Digestive Disorders, NHS Lothian, Edinburgh, UK

Introduction Fuji Intelligent Chromo Endoscopy (FICE) is a postprocessing image enhancement technique designed by Fuji for their video endoscopy systems. FICE is a method that takes the original white light image and separates the image into discrete light wavelengths, each setting has a different combination of three selected wavelengths (from 400 to $700 \mathrm{~nm}$ ), representing RGB, that are independently weighted, combined and superimposed on the white light image to produce the FICE image. Fuji currently provides 10 different settings. The aims of this project are: to evaluate the current FICE settings on images captured during upper GI endoscopy, and the creation of new oesophageal settings to simplify the selection and improve the application of FICE in the diagnosis of oesophageal pathology.

Methods We used a PC based FICE simulator, provided by Fuji, to process images offline, using the FICE settings. A series of images were captured during diagnostic endoscopies of various conditions (eg, varicies, Barrett's oesophagus) at various points in the oesophagus using the capture facilities on the Fuji endoscopy EPX-4400 processor. Using the FICE simulator, two new FICE settings were created to maximise the enhancement of the pathology while maintaining the anatomical colouring. For each RGB component, the selected wavelength was altered independently in $5 \mathrm{~nm}$ steps. Once the wavelength was fixed, the gain was altered to minimise artefacts and maximise enhancement. Forty images were selected from the series that covered various conditions, and were processed via the FICE simulator using the 10 standard and two new settings. The images were randomised for evaluation by five blinded endoscopists. Each endoscopist compared the original and FICE image and scored the degree of enhancement over the original image from 0 (no enhancement) to 5 (maximum). The highest score FICE settings were translated to the EPX-4400 processor for further clinical evaluation.

Results The oesophageal mucosa presented with two distinct shades of pink (eg, Light and Dark). Two settings were created, one for each mucosal shade (L1-Light and L2-Dark). From the 40 images $65 \%$ would be characterised as Light and 35\% Dark mucosa. Out of a possible enhancement score of 1000, the standard FICE
Settings (0-9) scored between 202 and 319, with settings 2 scoring the highest (319). The Lothian FICE settings L1 and L2 scored 463 and 387 respectively.

Conclusion In conclusion the L1/2 FICE settings were found to provide further enhancement compared with current FICE settings by improving the colour discrimination between normal and abnormal mucosa. FICE is a useful and flexible system with a lot of potential but still requires optimisation.

Competing interests $S$ Inglis Grant/Research Support from: Fuji provided software for Trial, S Alexandridis Grant/Research Support from: Fuji/lmotech Medical Provided funding for a Fellowship, J Plevris Grant/Research Support from: Fuji Provided Loan Equipment for trial.

\section{PWE-198 PROPOFOL ASSISTED ENDOSCOPY ON DAY-CASE ENDOSCOPY UNIT-“THE EXPERIENCE"}

doi:10.1136/gutjnl-2012-302514d.198

${ }^{1} \mathrm{~S}$ V Murugesan, ${ }^{2} \mathrm{M}$ Davies, ${ }^{1} \mathrm{~N}$ Haslam, ${ }^{1} \mathrm{H}$ L Smart, 'S Sarkar. "Department of Gastroenterology, Royal Liverpool and Broadgreen University Hospitals NHS Trust, Liverpool, UK; ${ }^{2}$ Department of Anaesthetics, Royal Liverpool and Broadgreen University Hospitals NHS Trust, Liverpool, UK

Introduction Patients intolerant of endoscopic procedure under conscious sedation subsequently have the procedure under a general anaesthetic. Provision of deep sedation (with propofol) has also been used in this setting.

Aim To assess the safety profile and patient satisfaction with deep sedation using propofol for endoscopic procedures in a dedicated deep sedation endoscopy list.

Methods Retrospective analysis of cases performed between June and December 2011. Cases were performed on a dedicated weekly "deep sedation" list supervised by a consultant anaesthetist in the endoscopy Unit. Anaesthetic records, case records and GI reporting tool (UNISOFT ${ }^{\circledR}$ ) were interrogated for data. Satisfaction scores (score 1=unsatisfactory; 5=fully satisfied) were recorded for patients, anaesthetists and endoscopists post procedure.

Results 40 patients, 19 (47.5\%) female with a median age of 53 years (range 18-80 years), underwent propofol assisted endoscopy. Mean ASA grade was 2 (range 1-3). 24 (60\%) had unsatisfactory endoscopy previously under conscious sedation for phobia, anxiety, pain (colonoscopy) and stricture requiring dilatation.

Procedures 16 (40\%) underwent radio frequency ablation of dysplastic lesion in Barrett's oesophagus $\left(\mathrm{HALO}^{\circledR}\right)$ and $14(35 \%)$ underwent colonoscopy, with the remainder undergoing ERCP $(n=3,7.5 \%)$, gastroscopy $(n=2,5 \%)$, ampullectomy $(n=2,5 \%)$, small bowel enteroscopy $(n=1,2.5 \%)$ and endoscopic ultrasound $(n=2,5 \%)$. Mean waiting time was 8 weeks. All procedures were successfully completed with mean duration of 33 min per procedure (range 10-70 min). Mean propofol dose administered was $333 \mathrm{mg}$ (range $41-1178 \mathrm{mg}$ ) and in addition fentanyl (mean dose $50 \mu \mathrm{g}$ ), midazolam (mean dose $1.5 \mathrm{mg}$ ), hyoscine hydrobromide $(20 \mathrm{mg})$ and intravenous paracetamol $(1 \mathrm{~g})$ were administered as required. No reversal agents were required for any of the procedures.

Adverse Events Overall rate was $10 \%$ and minor. Transient hypoxia ( $\mathrm{SpO} 2<90 \%$ ) in two patients, relieved with jaw thrust, one patient required an airway device and persistent hypotension in two patients required vasopressors. All patients were discharged as day-cases. There were no 7-day readmissions or 30-day mortality. Median satisfaction scores for the procedure were high for patients (5), for anaesthetists (5) and for endoscopists (5).

Conclusion Anaesthetic led propofol assisted endoscopy is safe in a day-case endoscopy unit and is associated with high satisfaction scores for patient, anaesthetist and endoscopists.

Competing interests None declared. 